\title{
Trichomyia quimbaya, una nueva especie de Trichomyiinae (Diptera: Psychodidae) de la Cordillera Central de Colombia
}

\author{
Eduar Elias Bejarano ${ }^{1,4}$, Alveiro Pérez-Doria ${ }^{1}$ \& Diana Sierra ${ }^{2,3}$ \\ ${ }^{1}$ Grupo de Investigaciones Biomédicas, Universidad de Sucre, \\ Cra. 14 No. 16 B-32, A.A. 406, Sincelejo, Colombia \\ ${ }^{2}$ Department of Microbiology, Immunology and Pathology, Colorado State University, Central receiving \\ 200 W, Lake Street Campus Delivery 1619, CO 80523, USA \\ ${ }^{3}$ Programa de Estudio y Control de Enfermedades Tropicales - PECET, Universidad de Antioquia, \\ Calle 62 No. 52-69, A.A. 1226, Medellín, Colombia \\ ${ }^{4}$ Autor de correspondencia: Eduar Elías Bejarano,e-mail: eduarelias@yahoo.com
}

BEJARANO, E.E., PÉREZ-DORIA, A. \& SIERRA, D. Trichomyia quimbaya, a new species of Trichomyiinae (Diptera: Psychodidae) from the Cordillera Central of Colombia. Biota Neotrop., 9(4): http://www. biotaneotropica.org.br/v9n4/en/abstract?article+bn02809042009.

\begin{abstract}
Trichomyia quimbaya sp. nov. is described and illustrated from a male specimen collected with a CDC light trap in the coffee region of the Cordillera Central of Colombia. It can be easily distinguished from others species of Trichomyia by the pair of strong digitiform prolongations of the gonocoxite, both of which end in large spines, with apex directed inwards.

Keywords: Diptera, Trichomyia, new species, Andean region, Colombia.
\end{abstract}

BEJARANO, E.E., PÉREZ-DORIA, A. \& SIERRA, D. Trichomyia quimbaya, una nueva especie de Trichomyiinae (Diptera: Psychodidae) de la Cordillera Central de Colombia. Biota Neotrop., 9(4): http:// www.biotaneotropica.org.br/v9n4/es/abstract?article+bn02809042009.

Resumen: Trichomyia quimbaya sp. nov. es descrita e ilustrada con base en un ejemplar macho recolectado con una trampa de luz CDC, en la zona cafetera de la Cordillera Central de Colombia. Ésta se diferencia fácilmente de otras especies de Trichomyia por las prolongaciones digitiformes prominentes del gonocoxito, que terminan en espinas largas con el ápice orientado hacia adentro.

Palabras-clave: Diptera, Trichomyia, especie nueva, región Andina, Colombia. 


\section{Introducción}

El género Trichomyia fue establecido para albergar a la especie paleártica Trichomyia urbica Haliday, 1839, descrita a partir del patrón de venas del ala. Este género permaneció monotípico hasta que Coquillet (1902) describió la especie neotropical Trichomyia cirrata Coquillet, 1902, con base en ejemplares hembras recolectados en México. Desde entonces se han descrito 75 especies de Trichomyia en América, 65 neotropicales, 4 neárticas y 6 fósiles de México.

El estudio de la subfamilia Trichomyiinae Tonnoir, 1922, en el Neotrópico, ha sido esporádico, aunque en la década pasada se renovó el interés por explorar las especies desconocidas para la ciencia, lo que permitió la descripción de 41 nuevos taxones (Wagner 1993, 1999, Wagner \& Masteller 1996, Quate 1996, 1999, Bravo 1999, 2000, 2001a,b,c, 2002, Alexander et al. 2001, Ibáñez-Bernal 2004). Las investigaciones sobre esta subfamilia se encuentran todavía en un nivel incipiente en Colombia, donde la presencia del taxón sólo se registró en el año 2006 (Bejarano 2006). No obstante, los muestreos preliminares indican que la diversidad del grupo en el territorio colombiano podría superar incluso a la hasta ahora observada en otros países neotropicales.

El propósito de este trabajo es describir una nueva especie de Trichomyia originaria de la Cordillera Central de Colombia.

\section{Material y Métodos}

El material tipo fue atraído con una trampa de luz CDC instalada a $1.5 \mathrm{~m}$ de altura, en la rama de un árbol, cerca de una corriente de aguas. El muestreo se llevó a cabo en el Jardín Botánico "Alejandro Humboldt" del municipio de Marsella, departamento de Risaralda, en la Cordillera Central de Colombia. El psicódido se aclaró durante 24 horas en lactofenol y se fijó en una lámina portaobjetos con bálsamo de Canadá. Las estimaciones morfométricas del ejemplar se realizaron con un micrómetro ocular en un microscopio triocular Carl Zeiss ${ }^{\circledR}$ Primo Star. La terminología usada para la descripción taxonómica corresponde, en parte, a la propuesta por McAlpine (1981). El holotipo reposa en la "Colección de Vectores y Hospedadores Intermediarios de Enfermedades Tropicales (VHET)" del PECET de la Universidad de Antioquia, en Medellín, Colombia.

\section{Resultados}

Trichomyia quimbaya Bejarano, Pérez-Doria \& Sierra, sp. nov.

Material tipo: Colombia, Rirasalda, Marsella (Jardín Botánico “Alejandro Humboldt”, 04 56' N y 75 44' O), holotipo: macho, trampa de luz CDC, 25.II.2004, Bejarano E. E. \& Sierra D. cols. (VHET).

Descripción: (todas las medidas están expresadas en micrómetros). Psicódido con la cabeza, el tórax y abdomen pigmentados de color marrón.

Cabeza: Cabeza mucho más ancha que larga, con una altura desde el vértice hasta la base del labroepifaringe de 313 y una anchura de 540. Vértice sin línea media, recubierto por cicatrices de setas entremezcladas con pilosidades, y con una hilera irregular de siete a ocho cicatrices grandes bordeando el margen del ojo. Clípeo recubierto por pelos pequeños y cicatrices de setas. Anchura del clípeo 65, con una altura de 80. Longitud del labro-epifaringe 75. Ojos compuestos, sin puente ocular, con una altura de 310. Distancia interocular 169, equivalente al diámetro de 8,5 facetas. Escapo y pedicelo subesféricos. Pedicelo con una longitud de 63 y anchura de 78. Flagelómeros basales con aspecto piriforme, articulados casi simétricamente (Figura 2). Longitud de los flagelómeros: I-118, II-108, III-115 y IV-120. Ascoides insertados al mismo nivel. Palpos maxilares constituidos por cuatro segmentos, recubiertos por setas foliáceas, y con el primer, segundo y tercer artejo más anchos que largos (Figura 1). Primer palpómero con 30 de largo y 45 de ancho. Segundo palpómero con 28 de largo y 43 de ancho. Tercer palpómero con 30 de largo y 34 de ancho, con tres setas espiniformes en el margen interno del ápice. Cuarto palpómero con una longitud de 65 y con cinco setas espiniformes en el borde interno de la mitad apical. Proporción de los palpómeros 1.0:0.93:1.0:2.17. Formula palpal (1.2.3).4. Primer y segundo segmento parcialmente fusionados y con un grupo de sensilas dispuestas en una depresión circular, sobre el lado interno.

Tórax: Alas con el ápice redondeado (Figura 5). Longitud del ala desde el inicio de la vena costa 1930, anchura máxima 864. Proporción entre la longitud y anchura máxima del ala 2,4. Longitud de las venas $R_{1} 1003, R_{2} 785, R_{3} 950, R_{4+5} 1410, M_{1} 1100$, $M_{2} 1010, M_{3} 1190, \mathrm{CuA}_{1} 930$. Venas $\mathrm{R}_{1}, \mathrm{R}_{4+5} \mathrm{y} \mathrm{CuA}_{1}$ engrosadas, con la vena $\mathrm{R}_{1}$ acentuadamente más esclerotizada (Figura 5). Bifurcaciones radial y media separadas por 191 . Vena Sc continua, alcanzando claramente las venas costa y $R_{1}$. Vena transversal $\mathrm{r}-\mathrm{m}$ ausente. Venas $\mathrm{R}_{2+3+4+5}$ y $\mathrm{M}_{2}$ discontinuas en la base. Vena $\mathrm{CuA}_{2}$ larga, insertándose al nivel de la bifurcación media. Halterios globosos, con una anchura máxima de 113, y longitud de 253 hasta el punto de inserción. Proporción entre la longitud y anchura máxima del halterio 2,24.

Abdomen: Abdomen y genitales con una longitud de aproximadamente 1219 , con los esternitos y tergitos revestidos por cicatrices de setas. Cercos subesféricos, recubiertos por setas espiniformes, superando el ápice de los gonostilos (Figura 4). Esternito 10 pequeño y redondeado (Figura 4). Gonocoxito con dos prolongaciones laterales digitiformes, gruesas, de tamaño desigual, cada una de las cuales da origen a una larga espina con la punta dirigida hacia el interior (Figura 3). De la base dorsal de los gonocoxitos se desprende el esternito 9 que presenta aspecto redondeado, relativamente translucido y revestido en forma dispersa por 28 setas delgadas (Figura 4), el cual distalmente parece unirse a los parámeros. Gonostilos esclerotizados y gruesos, con la punta orientada externamente (Figura 3). Parámeros esclerotizados, gruesos, curvos en la mitad, sin sobrepasar el ápice del edeago (Figura 3). Edeago de aspecto subrectangular, terminando en dos puntas robustas. La bifurcación del edeago está marcada por una pequeña estructura circular fuertemente esclerotizada (Figura 3), que es antecedida por un esclerito transversal de aspecto ovoide. Apodema eyaculador largo y recto, en visión dorso-ventral (Figura 3).

Etimología: El nombre de la especie hace referencia a la etnia precolombina Quimbaya, que habitó en el valle medio del Río Cauca, incluida la localidad donde se recolectó el holotipo.

\section{Discusión}

Hasta la fecha se han descrito 23 especies de Trichomyia en el Neotrópico, que poseen los dos primeros palpómeros parcialmente fusionados (Coquillett 1902, Rapp 1945, Barretto 1954a,b, Satchell 1956, Duckhouse 1972, Quate 1996, Wagner \& Masteller 1996, Bravo 2000, 2001a,b, 2002, Alexander et al. 2001). Sólo 10 de estos taxones exhiben un grupo de sensilas sobre el primer y segundo palpómero, T. cirrata Coquillett, 1902, T. brevitarsa (Rapp 1945), T. armata Barretto, 1954, T. brasilensis Satchell, 1956, T. buchholzi Wagner \& Masteller, 1996, T. quatei Bravo, 2001, T. nocturna Bravo, 2001, T. fluminensis Bravo, 2001, T. onorei Bravo, 2002 y T. queirozi Bravo, 2002, característica que también se observa en T. quimbaya sp. nov. La nueva especie se diferencia fácilmente de todas las anteriores por 

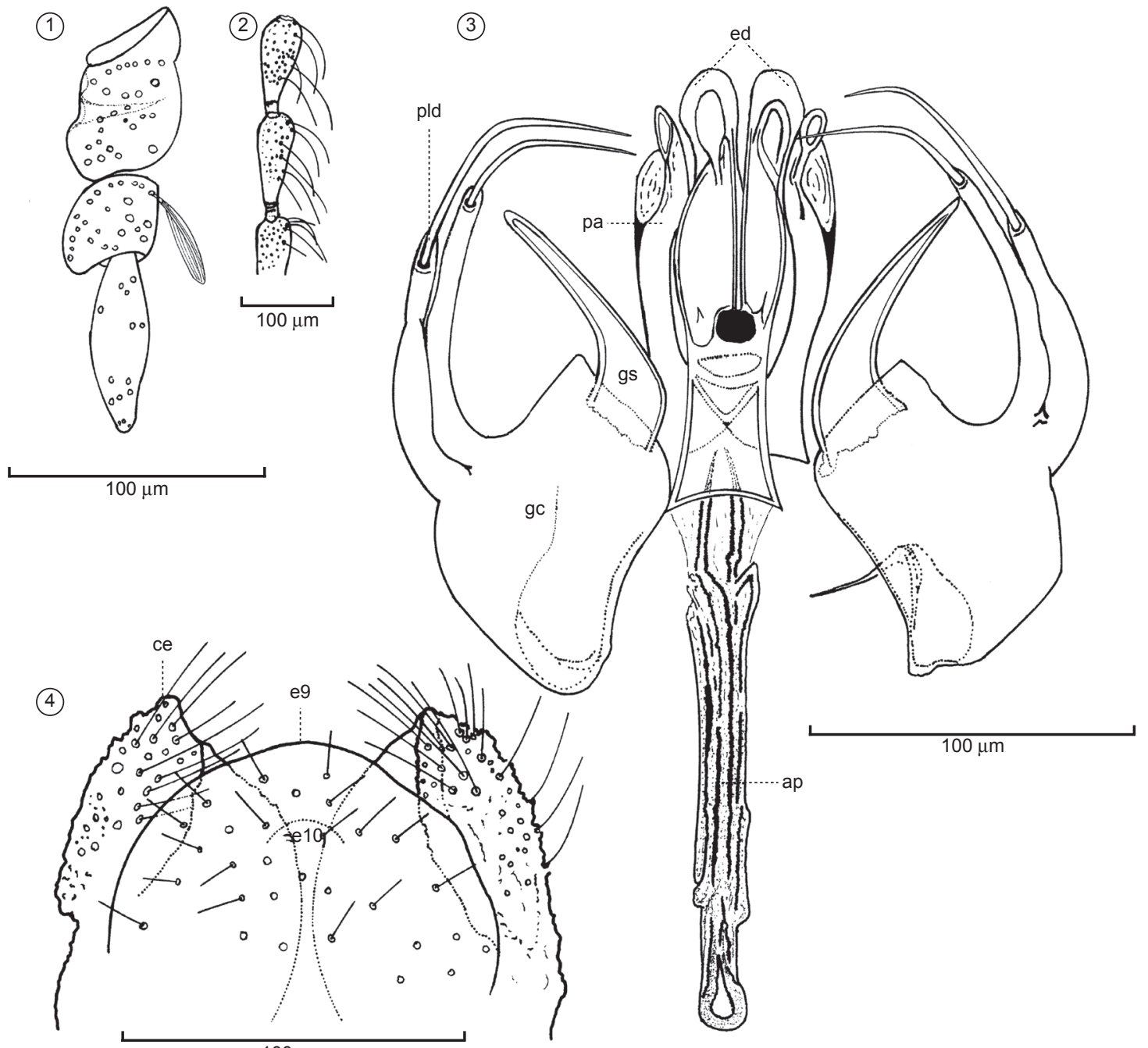

(4)

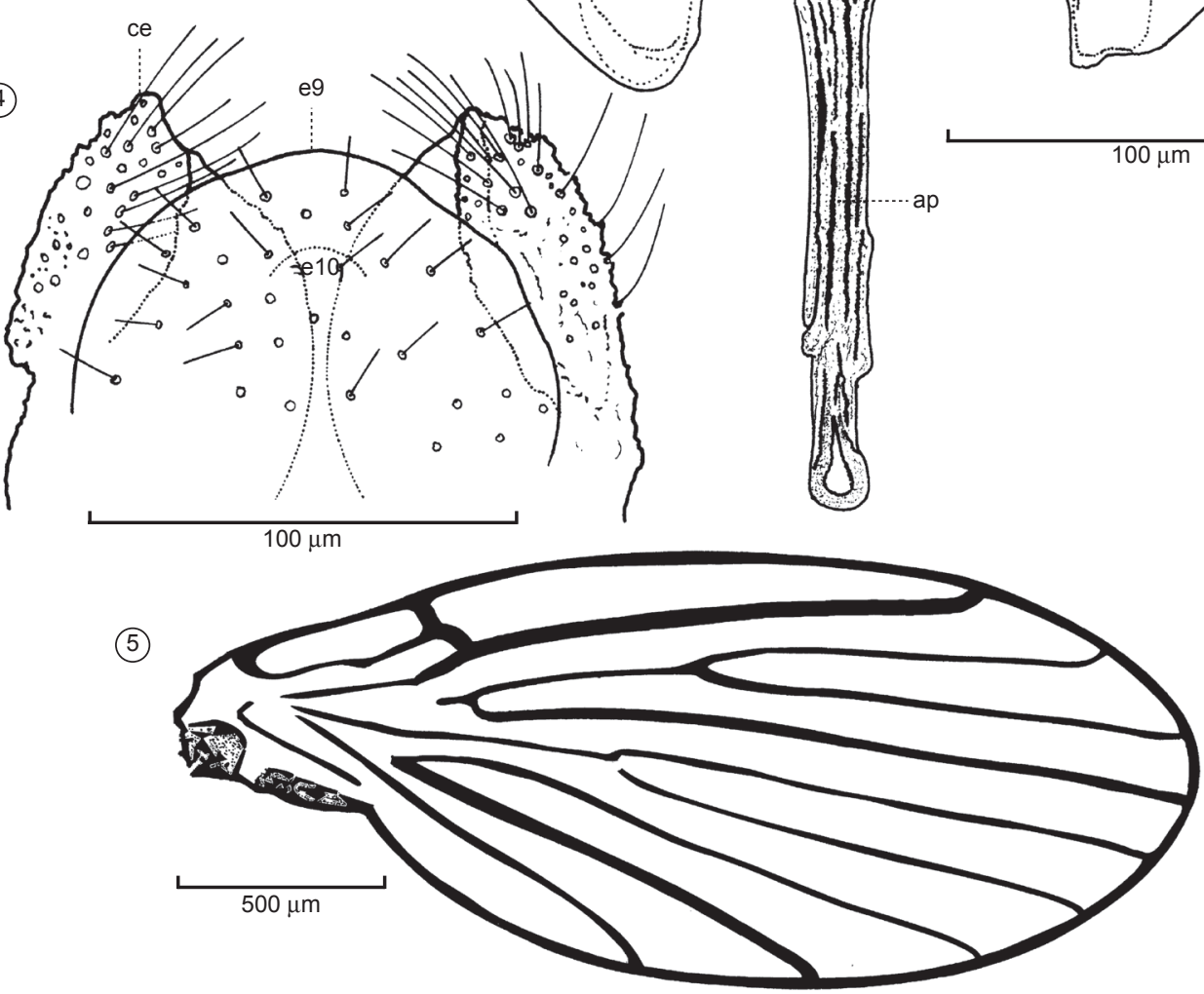

Figuras 1-5. Trichomyia quimbaya Bejarano, Pérez-Doria \& Sierra, sp. nov., holotipo macho. 1) palpómeros, escala $100 \mu$ m; 2) flagelómeros basales, escala $100 \mu \mathrm{m}$; 3) genitalia en visión dorso-ventral, escala $100 \mu \mathrm{m}$; 4) cercos, y esternitos 9 y 10 en visión dorso-ventral, escala 100 $\mu \mathrm{m}$; y 5) ala, escala 500 $\mu \mathrm{m}$. (ed = edeago; $\mathrm{gs}=$ gonostilo; $\mathrm{gc}=$ gonocoxito; $\mathrm{pld}=$ prolongación lateral digitiforme del gonocoxito; $\mathrm{pa}=$ parámero; $\mathrm{ap}=$ apodema eyaculador; ce = cerco; e9 = esternito 9; e10 = esternito 10).

Figures 1-5. Trichomyia quimbaya Bejarano, Pérez-Doria \& Sierra, sp. nov., male holotype. 1) palpomeres, scale bar $100 \mu \mathrm{m}$; 2) basal flagellomeres, scale bar $100 \mu \mathrm{m}$; 3) genitalia in dorso-ventral view, scale bar $100 \mu \mathrm{m}$; 4) cerci and $9^{\text {th }}$ and $10^{\text {th }}$ sternite in dorsal-ventral view, scale bar $100 \mu \mathrm{m}$; and 5) wing, scale bar $500 \mu \mathrm{m}$. (ed = aedeagus; gs = gonostyle; gc = gonocoxite; pld = lateral prolongation digitiform of the gonocoxite; pa = paramere; ap = ejaculatory apodem; ce $=$ cercus; $\mathrm{e} 9=9^{\text {th }}$ sternite; $\mathrm{e} 10=10^{\text {th }}$ sternite $)$.

las prolongaciones digitiformes prominentes del gonocoxito, que terminan en largas espinas con el ápice orientado hacia adentro, la forma y esclerotización de los gonostilos, y el aspecto del complejo del edeago, con el largo apodema eyaculador.
T. brevitarsa, T. nocturna y T. fluminensis, pertenecen al subgénero Opisthotrichomyia Bravo, 2001, que se distingue por poseer un lóbulo interno en el gonocoxito, recubierto por setas largas y delgadas (Bravo 2001a), lo que permite separar las tres especies de 
T. quimbaya sp. nov. De otro lado, T. armata, T. brasilensis y T. quatei, constituyen un taxón natural caracterizado por la presencia de una prolongación dorsal en el gonocoxito, revestida por setas gruesas y largas que se distribuyen en el borde interno de este apéndice (Bravo 2001c), rasgo que las diferencia de la nueva especie. Así mismo, T. onorei y T. queirozi son especies muy cercanas entre sí, con el gonocoxito desprovisto de prolongaciones, lo cual las distingue de T. quimbaya sp. nov. Por su parte, T. buchholzi presenta un solo apéndice completamente curvo en el gonocoxito y un apodema eyaculador corto, a diferencia de la nueva especie que exhibe dos prolongaciones laterales en el gonocoxito y un apodema eyaculador largo.

Por sus particularidades morfológicas, T. quimbaya sp. nov. no puede agruparse en los subgéneros de Trichomyia hasta ahora reconocidos. No obstante, la descripción de esta nueva especie a partir de un solo ejemplar macho se justifica por los rasgos notoriamente distintivos que exhibe el espécimen hallado y el enorme vacío existente en el conocimiento de este grupo de insectos en la región andina. Se hace necesario entonces continuar con la descripción de los Trichomyiinae neotropicales, para luego establecer las relaciones de afinidad entre las distintas especies del Nuevo y Viejo Mundo.

\section{Referencias}

ALEXANDER, B., FREITAS, J.M. \& QUATE, L.W. 2001. Some Psychodidae (Diptera) from Atlantic forest in South-Eastern Brazil, with descriptions of Trichomyia dolichopogon sp. nov. and Trichomyia riodocensis sp. nov. Braz. J. Biol. 61(3):467-474.

BARRETTO, M.P. 1954a. Novas espécies de Trichomyia Hal. do Brasil (Diptera, Psychodidae). Folia Clin. Biol. 21:127-137.

BARRETTO, M.P. 1954b. Sobre o gênero Eubonetia Vargas e Diaz-Nájera com a descrição de novas espécies do Brasil (Diptera, Psychodidae). Rev. Bras. Entomol. 2:81-195.

BEJARANO, E.E. 2006. Subfamilia Trichomyiinae (Psychodidae) en el territorio continental e insular colombiano. Act. Biol. Colom. 11(1): $37-41$.

BRAVO, F. 1999. Septemtrichomyia, subgênero novo de Trichomyiinae Neotropical (Diptera, Psychodidae). Rev. Bras. Entomol. 43(1/2):1-7.

BRAVO, F. 2000. Descrição de uma espécie de Trichomyia (Diptera, Psychodidae) do sudeste brasileiro, com comentários sobre a genealogia do gênero. Acta Biol. Leopoldensia. 22(2):185-192.

BRAVO, F. 2001a. Opisthotrichomyia, subgênero novo de Trichomyiinae (Diptera, Psychodidae) e descrição de três novas espécies do Brasil. Sitientibus, Sér. Ciênc. Biol. 1(1):50-55.

BRAVO, F. 2001b. Sete novas espécies de Trichomyia (Diptera, Psychodidae) da Mata Atlântica do Nordeste do Brasil. Sitientibus, Sér. Ciênc. Biol. 1(2):121-130.
BRAVO, F. 2001c. Trichomyia quatei (Diptera, Psychodidae), uma nova espécie do nordeste brasileiro. Acta Biol. Leopoldensia. 23(1):31-37.

BRAVO, F. 2002. Novas espécies de Trichomyia (Diptera, Psychodidae) da Mata Attântica da Bahia, nordeste do Brasil. Iheringia, Sér. Zool. 92(3):57-67.

COQUILLETT, D.W. 1902. New Orthorrhaphous Diptera from Mexico and Texas. J. New York Entomol. Soc. 10:136-141.

DUCKHOUSE, D.A. 1972. Psychodidae (Diptera, Nematocera) of South Chile, subfamilies Sycoracinae and Trichomyiinae. Trans. R. Entomol. Soc. Lond. 124(3):231-268.

IBÁÑEZ-BERNAL, S. 2004. Notes on the known species of Trichomyia Haliday of Mexico, with the establishment of a synonymy and the description of a new species (Diptera: Psychodidae). Zootaxa. 523: $1-14$.

McALPINE, J.F. 1981. Morphology and terminology - adults. In Manual of Neartic Diptera (J.F. Mcalpine, B.V. Peterson, G.E. Shewell, H.J. Teskey, J.R. Vockeroth \& D.M. Wood, eds.). Research Branch, Agriculture Canada, Ottawa, p. 9-63. (Monograph 27, v.1)

QUATE, L.W. 1996. Preliminary taxonomy of Costa Rican Psychodidae (Diptera), exclusive of Phlebotominae. Rev. Biol. Trop. 44(Supl.1): $1-81$.

QUATE, L.W. 1999. Taxonomy of Neotropical Psychodidae (Diptera) 3. Psychodines of Barro Colorado Island and San Blas, Panama. In Contributions to the knowledge of Diptera. (J.F. Burger, ed.). Associated Publishers, Gainesville, Florida, p. 405-441. (Memoirs on entomology, International, v.14)

RAPP, W.F. 1945. New Psychodidae from Barro Colorado Island. J. New York Entomol. Soc. 53:309-311.

SATCHELL, G.H. 1956. On the genus Trichomyia Haliday (Diptera: Psychodidae), with descriptions of four new species. Proc. R. Ent. Soc. Lond. B. 25:147-156.

WAGNER, R. \& MASTELLER, E.C. 1996. New moth flies (Diptera: Psychodidae) and a key to species from Puerto Rico. Proc. Entomol. Soc. Wash. 98(3):450-464.

WAGNER, R. 1993. On a collection of Psychodidae (Diptera) by Dr. L. Botosaneanu from some Caribbean Islands. Aquatic Insects. 15(2): 109-127.

WAGNER, R. 1999. Psychodidae from the Dominican Republic: records and descriptions of new species (Insecta: Diptera). J. Kans. Entomol. Soc. 72(2):233-245. 